\title{
Robust Time-of-Arrival Self Calibration with Missing Data and Outliers
}

\author{
Kenneth Batstone, Magnus Oskarsson and Kalle Åström \\ Centre for Mathematical Sciences, \\ Lund University, \\ Lund, Sweden \\ kenneth_john.batstone@math.lth.se, \{magnuso,kalle\}@maths.lth.se
}

\begin{abstract}
The problem of estimating receiver-sender node positions from measured receiver-sender distances is a key issue in different applications such as microphone array calibration, radio antenna array calibration, mapping and positioning using ultrawideband and mapping and positioning using round-trip-time measurements between mobile phones and Wi-Fi-units. Thanks to recent research in this area we have an increased understanding of the geometry of this problem. In this paper, we study the problem of missing information and the presence of outliers in the data. We propose a novel hypothesis and test framework that efficiently finds initial estimates of the unknown parameters and combine such methods with optimization techniques to obtain accurate and robust systems. The proposed systems are evaluated against current state-of-the-art methods on a large set of benchmark tests. This is evaluated further on Wi-Fi roundtrip time and ultra-wideband measurements to give a realistic example of self calibration for indoor localization.
\end{abstract}

\section{INTRODUCTION}

In this paper we present new research on robust methods for time-of-arrival (TOA) self-calibration problem with missing data and outliers. This is then applied to Wi-Fi round-trip time (RTT) and ultra-wideband indoor localization measurements to provide realistic examples. The TOA self-calibration problem is the problem of determining the positions of a number of receivers and transmitters given receiver-transmitter distances. In this problem, there is no assumption that there exists a subset of sensors (anchors) whose locations are known. Hence it is closely related to the anchor-free sensor network localization problem [1] but differs, since the transmitters or receivers are independent of each other. Our problem structure corresponds to a bipartite graph, unlike [1] where the network structure is a general graph. The TOA problem also has certain similarities with the problem of determining a set of points given all inter-point distances, which is usually solved using multi-dimensional scaling [2]. Such problems are of general interest in visualization and analysis of large datasets (e.g. DNA data), in machine learning and for many geometric problems. The TOA self-calibration problem is important for node calibration problems for a variety of different media, e.g. (i) microphone arrays (given recordings of sounds emitted at unknown locations, to microphones at unknown positions, determine both sound emission positions and microphone locations), similarly (ii) ultra-sound, (iii) radio (Ultra Wide

Thanks to MAPCI and ELLIIT for funding.
Band), (iv) Wi-Fi (Using signal strength) and (v) Wi-Fi roundtrip time measurements (RTT).

Anchor-free sensor network calibration with time-of-arrival measurements has been investigated in a number of studies. Graph rigidity was explored in [1] to find a fold-free graph embedding. The solution was then refined using mass-spring based optimization. In [3], a semi-definite programming formulation and solution was proposed for TOA measurements, with or without anchors. Both of these methods are general for any solvable network structure. Another line of work has focused on sensor networks with bipartite structure, that appear in various applications mentioned in the previous paragraph. For this special type of bipartite network structure, one also aims to identify and solve the minimal problem, i.e. minimal number of receivers and transmitters required for the problem to be well-defined (or solvable). Note that for this problem, the roles of receivers and transmitters are equivalent. Therefore, when discussing minimal cases, the number of sensors required for receivers and transmitters are interchangeable. The minimal cases were studied in [5], where solutions to the minimal case of 3 transmitters and 3 receivers in the plane are given. The minimal problems for the $3 \mathrm{D}$ case are given in [6]. The minimal number of receivers and senders are $(4,6),(5,5)$ and $(6,4)$ respectively. There are in general 38, 42 and 38 solutions respectively for the three types of problems. However, no practical methods for general 3D positions are given. There are a few results on algorithms for actually determining the positions from distances, most notably [7], [8]. In [9], a non-minimal linear solution to the 3D TOA self-calibration problem is derived for 10 (4) receivers and 4 (10) transmitters. In [10], [11] a solution is given to the TOA self-calibration problem, if one may additionally assume that one of the receivers coincides with the position of one of the transmitters. The minimal cases for far field approximation were first studied in [12] and later refined in [13]. In far field approximation, the distances between the transmitters and receivers are assumed to be considerably larger than those between receivers. The solutions based on the far field approximation can be utilized to initialize the original TOA problem.

Studying these minimal cases is of theoretical importance 
and further more essential when developing fast and stable algorithms based on robust estimation methods like RANSAC [14], in the presence of outliers in the measurements. As will be shown in the following sections, one important part of our system exploits that the so-called compaction matrix should have a certain rank. Low rank matrix factorization has a long standing history. Truncating the singular value decomposition of the measurement matrix has been shown to give the optimal solution under the $l^{2}$-norm for complete data, see [15]. The work in [16] was the first to consider missing data. Robustness to outliers has been considered in [17], [18], [19], [20]. Most methods mentioned above are based on alternating optimization and are prone to get trapped in local minima. Recently, several works [21], [22], [23] re-formulate the problem to minimize the convex surrogate of the rank function, that is, the nuclear norm. For applications with a given fixed rank, the nuclear norm based methods usually perform inferior to the bilinear formulation-based methods [24]. A few recent works [25], [26] also explore the idea to divide the whole matrix into overlapping sub-blocks and combine the sub-block solutions. Minimal cases for low rank matrix factorization, for missing data, were investigated in [27].

Indoor localization is a currently a key issue, from needing to know the location of objects using Ultra-Wide Band beacons to finding the location of mobile phones with Wi-Fi when a GPS signal cannot be acquired. This is prevalent indoors and in build up areas such as New York, which is known as the "urban canyon" problem. Methods, like the one proposed, could be useful in solving these real world problems.

\section{BASIC GEOMETRY}

We will now describe the basic underlying geometry of our problem. Let $\mathbf{r}_{i}, i=1, \ldots, m$ and $\mathbf{s}_{j}, j=1, \ldots, n$ be the spatial coordinates of $m$ receivers (e.g. microphones) and $n$ transmitters (e.g. sound events), respectively. For measured time of arrival $t_{i j}$ from transmitter $\mathbf{r}_{i}$ and receiver $\mathbf{s}_{j}$, we have $v t_{i j}=\left\|\mathbf{r}_{i}-\mathbf{s}_{j}\right\|_{2}$ where $v$ is the speed of measured signals and $\|\cdot\|_{2}$ is the $l^{2}$-norm. The speed $v$ is assumed to be known and constant. We further assume that we, at each receiver can distinguish which transmitter $j$ each event is originating from. This can be done e.g. if the signals are temporally separated or using different frequencies. We will in the following work with the distance measurements $d_{i j}=v t_{i j}$. It is quite common that such data contains both missing data (not every sound event is detected at every microphone) and outliers (e.g. due to errors in the matching process). The TOA calibration problem can then be defined as follows,

Problem 1: (Time-of-Arrival Self-Calibration) Given absolute distance measurements

$$
d_{i j}=\left\|\mathbf{r}_{i}-\mathbf{s}_{j}\right\|_{2}+\epsilon_{i, j},
$$

for a subset $W \subset I$ of all the receiver-transmittor index pairs $I=\{(i, j) \mid i=1, \ldots m, j=1, \ldots, n\}$ determine receiver positions $\mathbf{r}_{i}, i=1, \ldots, m$ and transmitter positions $\mathbf{s}_{j}, j=$ $1, \ldots, n$. Here the errors $\epsilon_{i, j}$ are assumed to be either inliers, in which case the errors are small $\left(\epsilon_{i, j} \in N(0, \sigma)\right)$ or outliers, in which case the measurements are way off.
Here we will use the set $W_{i}$ for the indices $(i, j)$ corresponding to the inlier measurements and $W_{o}$ for the indices corresponding to the outlier set.

We will now show how the TOA calibration problem is solved generally. From many types of media, a transmitterreceiver distance will be acquired, $d_{i j}$. Since this can be assumed to be real and positive, it can be squared as follows,

$$
d_{i j}^{2}=\left(\mathbf{r}_{i}-\mathbf{s}_{j}\right)^{T}\left(\mathbf{r}_{i}-\mathbf{s}_{j}\right)=\mathbf{r}_{i}^{T} \mathbf{r}_{i}+\mathbf{s}_{j}^{T} \mathbf{s}_{j}-2 \mathbf{r}_{i}^{T} \mathbf{s}_{j} .
$$

The problem is then reformed according to the following invertible linear combinations of $d_{i j}^{2}$ :

$$
\mathbf{B}=\left(\begin{array}{cccc}
d_{11}^{2} & d_{12}^{2}-d_{11}^{2} & \ldots & d_{1 n}^{2}-d_{11}^{2} \\
d_{21}^{2}-d_{11}^{2} & & \hat{\mathbf{B}} & \\
\ldots & &
\end{array}\right)
$$

where the compaction matrix $\hat{\mathbf{B}}$ is an $(m-1) \times(n-1)$ matrix with entries as $\hat{B}_{i j}=\frac{d_{i, j}^{2}-d_{i 1}^{2}-d_{1 j}^{2}+d_{11}^{2}}{-2}$, with $i=$ $2, \ldots m$ and $j=2, \ldots, n$. The other elements in $\mathbf{B}$ are used as constraints for the solution.

The factorization can then be interpreted as follows. Let $\mathbf{R}_{i}=\left[\left(\mathbf{r}_{i}-\mathbf{r}_{1}\right)\right]$ and $\mathbf{S}_{j}=\left[\left(\mathbf{s}_{j}-\mathbf{s}_{1}\right)\right]$. Here $\hat{\mathbf{B}}=\mathbf{R}^{T} \mathbf{S}$ with $\mathbf{R}_{i}$ as columns of $\mathbf{R}$ and $\mathbf{S}_{j}$ as columns of $\mathbf{S}$. Since we assume that $\mathbf{R}$ and $\mathbf{S}$ are in a $3 \mathrm{D}$ affine space, the matrix $\hat{\mathbf{B}}$ has rank 3 at most. This also implies that in order to solve the problem, it is required that $m \geq 4$ and $n \geq 4$. By factorizing $\hat{\mathbf{B}}$, we can compute the vectors to all receivers and transmitters from unknown initial/reference positions $\left(\mathbf{r}_{1}\right.$ and $\mathbf{s}_{1}$ ).

By fixing $\mathbf{r}_{1}$ at the origin and $\mathbf{s}_{1}$ as a vector from the origin, in terms of an affine transformation matrix $\mathbf{L}$ and vector $\mathbf{b}$, the problem is reformulated as follows,

$$
\begin{aligned}
& \mathbf{r}_{1}=\mathbf{0}, \mathbf{s}_{1}=\mathbf{L} \mathbf{b}, \mathbf{r}_{i}=\mathbf{L}^{-T} \tilde{\mathbf{R}}_{i}, i=2 \ldots m, \\
& \mathbf{s}_{j}=\mathbf{L}\left(\tilde{\mathbf{S}}_{j}+\mathbf{b}\right), j=2 \ldots n,
\end{aligned}
$$

where $\tilde{\mathbf{R}}=\mathbf{L}^{T} \mathbf{R}, \tilde{\mathbf{S}}=\mathbf{L}^{-1} \mathbf{S}$, and hence $\hat{\mathbf{B}}=\tilde{\mathbf{R}}^{T} \mathbf{L}^{-1} \mathbf{L} \tilde{\mathbf{S}}=$ $\mathbf{R}^{T} \mathbf{S}$.

Using this parametrization, the equations from matrix $\mathbf{B}$, (3) become

$$
\begin{aligned}
d_{11}^{2} & =\left(\mathbf{r}_{1}-\mathbf{s}_{1}\right)^{T}\left(\mathbf{r}_{1}-\mathbf{s}_{1}\right)=\mathbf{s}_{1}^{T} \mathbf{s}_{1}=\mathbf{b}^{T} \mathbf{L}^{T} \mathbf{L} \mathbf{b} \\
& =\mathbf{b}^{T} \mathbf{H}^{-1} \mathbf{b}, \\
d_{1 j}^{2}-d_{11}^{2} & =\mathbf{s}_{j}^{T} \mathbf{s}_{j}-\mathbf{s}_{1}^{T} \mathbf{s}_{1}=\tilde{\mathbf{S}}_{j}^{T} \mathbf{L}^{T} \mathbf{L} \tilde{\mathbf{S}}_{j}+2 \mathbf{b}^{T} \mathbf{L}^{T} \mathbf{L} \tilde{\mathbf{S}}_{j} \\
& =\tilde{\mathbf{S}}_{j}^{T} \mathbf{H}^{-1} \tilde{\mathbf{S}}_{j}+2 \mathbf{b}^{T} \mathbf{H}^{-1} \tilde{\mathbf{S}}_{j}, \\
d_{i 1}^{2}-d_{11}^{2} & =\mathbf{r}_{i}^{T} \mathbf{r}_{i}-2 \mathbf{r}_{i}^{T} \mathbf{s}_{1}=\tilde{\mathbf{R}}_{i}^{T}\left(\mathbf{L}^{T} \mathbf{L}\right)^{-1} \tilde{\mathbf{R}}_{i}-2 \mathbf{b}^{T} \tilde{\mathbf{R}}_{i} \\
& =\tilde{\mathbf{R}}_{i}^{T} \mathbf{H} \tilde{\mathbf{R}}_{i}-2 \mathbf{b}^{T} \tilde{\mathbf{R}}_{i},
\end{aligned}
$$

where the symmetric matrix $\mathbf{H}=\left(\mathbf{L}^{T} \mathbf{L}\right)^{-1}$. With this parameterization, there are in total 9 unknowns ( 6 and 3 unknowns for $\mathbf{H}$ and $\mathbf{b}$, respectively), and hence a solution can be found. Since this solution has its own coordinate system, with prior knowledge this can be transformed back to the original coordinate system. 


\section{NON-LINEAR OPTIMIZATION APPROACHES}

In the development of the different systems for robust estimation, we use several different local optimization techniques. In particular we use methods for local optimization of the type

$$
\min _{\mathbf{r}, \mathbf{s}} \sum_{(i, j) \in \tilde{W}} f\left(d_{i, j}-\left\|\mathbf{r}_{i}-\mathbf{s}_{j}\right\|_{2}\right),
$$

where $f(r)$ is chosen to be (i) $f(r)=r^{2}$ ( $l^{2}$-norm), (ii) $f(r)=|r|\left(l^{1}\right.$-norm) or (iii) $f(r)=\min \left(r^{2}, T\right)$ (truncated $l^{2}$-norm). If the subset $\tilde{W}$ of the measurements contains no outliers and if the starting point is good, then the $l^{2}$-norm can give good estimates. Optimizing using the $l^{1}$-norm is less sensitive to the subset $\tilde{W}$ containing outliers, but still requires a reasonably good starting point to converge to a good solution. Local optimization of the truncated $l^{2}$-norm is even more sensitive to having a good starting point. Nevertheless, these local optimization techniques are important components for designing robust systems.

\section{OBTAINING INITIAL ESTIMATES}

Finding the optimal solution to problem 1, in the presence of outliers and missing data is a highly non-convex problem. We are thus dependent on finding good initial starting solutions, for the optimization methods from the previous section to work. We will in this section describe the different initialization methods that we have used in our experiment. In the next section we will describe our main contribution to the initialization problem.

Arguably, the most straight-forward way to initialize a solution, is to simply randomly place all receivers and senders within some space. This usually gives poor initial estimates, and the local optimization will be prone to get stuck in local minima. A slight improvement to this idea, is to use multiple restarts and optimize from each initial position, and then in the end choose the best solution.

Another way of initializing, that we have explored, is using the rank constraint on the compaction matrix. Here one can use many existing methods for doing the low rank matrix factorization. One important draw-back of these methods, is that we need to have at least one row and one column of the data matrix completely known, and without outliers. The last criteria is of course hard to check. If all data is known, the optimal low rank factorization is given by singular value decomposition (SVD) of the data matrix. A heuristic for handling missing data, is simply to fill in the missing data with some random values that follow the statistics of the other known measurements. One can then use SVD to obtain an initial estimate. This can be used directly to find the solution to the original problem as described in section II. Alternatively, the initial low rank matrix factorization can be refined using the Wiberg algorithm, [16].

\section{RANDOM SAMPLING PARADIGM}

The RANSAC or hypothesize and test paradigm, has proven to be useful in situations where there are outliers in the data, [14]. In this paradigm, a subset of the data is used to estimate

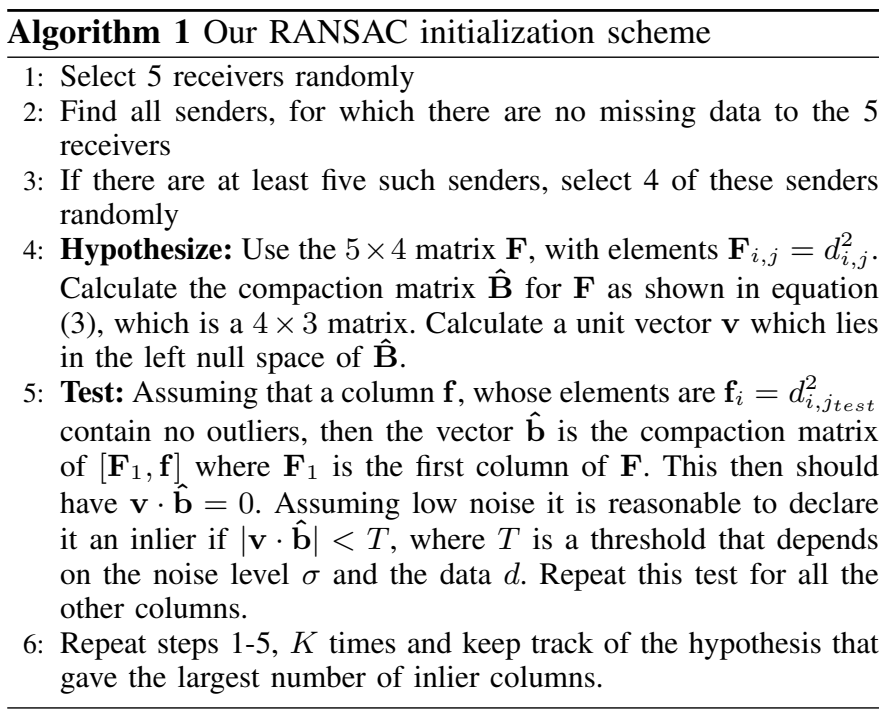

the unknown parameters. The remainder of the data is then used to verify or falsify the parameters. This is typically repeated a fixed number of iterations. The parameters that give the largest number of inliers are then usually used as an initial estimate for the subsequent non-linear optimization of the parameters.

For Problem 1, there are several ways one could implement the hypothesis and test paradigm. One idea would be to use efficient algorithms for determining receiver and sender positions from minimal data, [6]. Although this solver and the test is relatively fast, we propose an alternative to this approach. The main idea is to find a fast way to hypothesize and test. We will use the rank constraints of the compaction matrix to do this. Our method is described in Algorithm 1.

\section{EXPERIMENTAL EVALUATION}

For the experimental evaluation, we generated a series of scenarios with different missing data ratio, outlier data ratio and different levels of inlier noise. For simplicity we have kept the number of receivers $(m)$ and senders $(n)$ fixed at $m=30$ and $n=30$. We also fixed the room size to be $10 \times 10 \times 3$ meters and placed the ground truth positions of the receivers and senders randomly in this box using a uniform distribution. Finally the errors $\epsilon_{i, j}$ for outliers were randomly drawn uniformly in the intervals $[-1.2,-0.4]$ and $[0.4,1.2]$ meters. This means that for low levels of noise there is a relatively clear difference between inlier and outlier distributions. For higher levels of noise the inlier and outlier distributions will start to overlap considerably. Notice, however, that it is difficult to determine which measurements are inliers by simply studying the distance measurements.

For each setting we generate a number of synthetic scenarios where the ground truth position of the receivers and senders are placed randomly as described above. A random subset $W$ out of the $m n$ measurements in $I$ are chosen so that $|W| /|I| \approx$ $1-\sigma_{o}$. Then a random subset $W_{i} \subset W$ of inlier measurements are chosen so that $\left|W_{i}\right| /|W| \approx 1-\sigma_{i}$. The measurements $d_{i, j}$ 

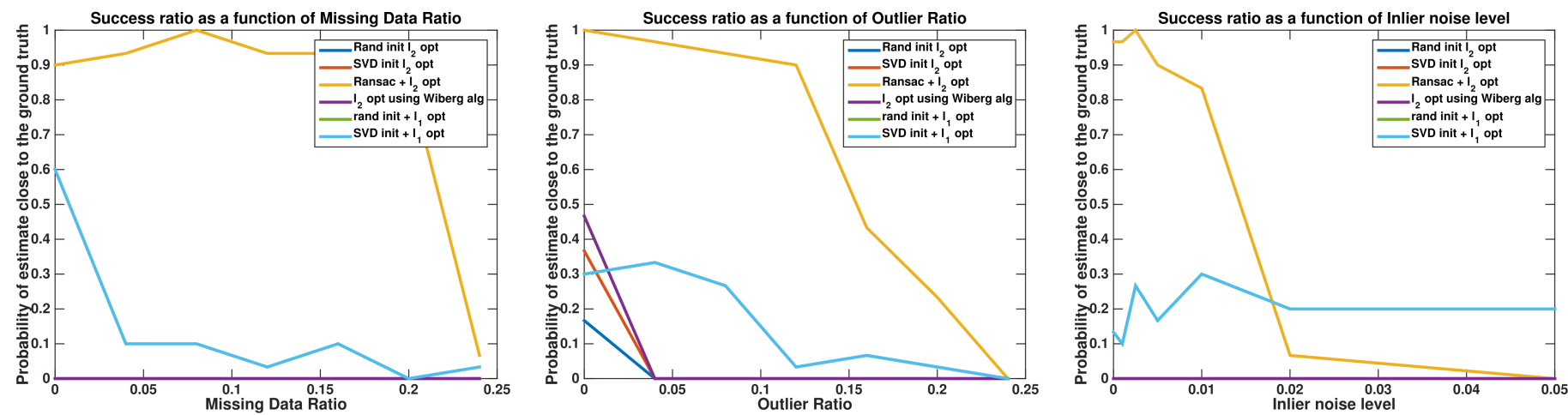

Fig. 1. Comparison of success ratios for a number of tested systems, as functions of (from left to right) missing data ratio, outlier ratio and inlier noise level respectively.

are generated according to (1) with added noise according to the inlier model for $(i, j) \in W_{i}$ and according to the outlier model for $(i, j) \in W_{o}=W \backslash W_{i}$.

Each synthetic dataset $d$ is then tested against each method. Here we tested the following systems

- Ransac $+l^{2}$ opt: Our proposed framework with initial estimate using RANSAC as described in Section 5, followed by $l^{2}$ optimization on detected inlier set.

- SVD Init $+l^{1}$ opt: Initial estimate using SVD as described in Section 4, followed by $l^{1}$ optimization.

- Rand Init $+l^{1}$ opt: Initial estimate using random placement as described in Section 4, followed by $l^{1}$ optimization

- $l^{2}$ opt using Wiberg alg: Initial estimate using SVD as described in Section 4, followed by rank 3 factorization using Wiberg algorithm followed by $l^{2}$ optimization.

- SVD Init $+l^{2}$ opt: Initial estimate using SVD as described in Section 4, followed by $l^{2}$ optimization

As can be seen in Figure 1, the proposed method outperforms the other methods in terms of robustness against outliers and missing data. For increased levels of noise the $l^{1}$ optimization methods degrades more gracefully.

For further experimental evaluation, our method was tested on real data measurements. In Figure 2, we conducted a roundtrip time Wi-Fi experiment in 2D using 4 Nexus 6 phones as anchors and one phone as a transmitter. These phones come with a IEEE 802.11.mc Wi-Fi standard, which produces round-trip time measurements in metres. The experimental environment that was chosen was a large open space in an office block (Ideon Alfahuset, Lund, Sweden) with dimensions $\sim 12 \times 18 \mathrm{~m}$.

For the experiment, a ground truth was measured for all the anchor points and the path of the moving source every $0.5 \pm$ $0.005 \mathrm{~m}$ with a tape measure. The experiment was conducted by walking the predefined path with the transmitter. In our experiment, our proposed framework does not require prior knowledge about the positions for any of the anchors or source locations nor the number of anchors and source locations. The only requirement is that the number of anchors and source locations satisfy the requirement of the minimal solver, for this $2 \mathrm{D}$ case $(3,3)$.

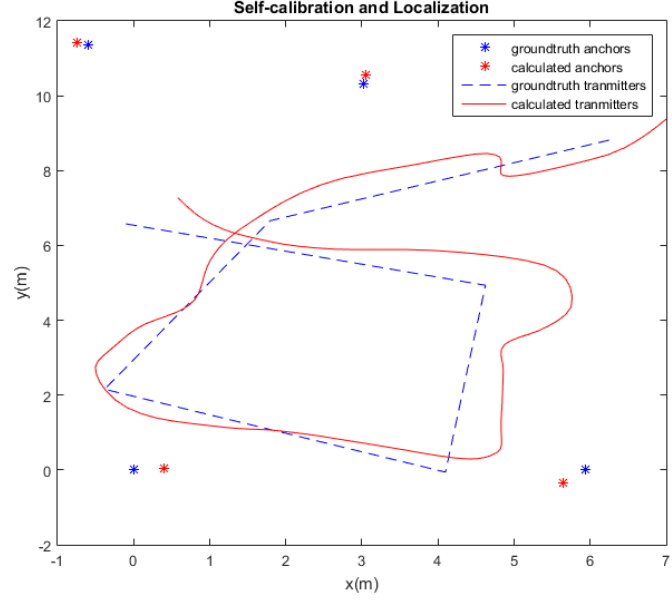

Fig. 2. This figure illustrates the estimated anchor positions and the source positions. This is overlaid on the ground truth anchor positions and predefined path.

A fixed number of iterations was used; 20 iterations for the initial selection of 3 receivers and senders, then a further 140 iterations to extend the number of columns and rows. The tolerance was set to $T=3$ for the initial selection and extension of columns then reduced to $T=2$ for the extensions of the rows.

Once the initial values have been estimated, it undergoes $l^{2}$ optimization on the inlier set. We also added a smoothness prior in the optimization. This prior is based on minimizing acceleration, according to

$$
\operatorname{res}_{a}=\frac{1}{\sigma_{a}^{2}} \sum_{j=2}^{n-1}\left\|\mathbf{s}_{j-1}-2 \mathbf{s}_{j}+\mathbf{s}_{j+1}\right\|_{2}^{2},
$$

where $\sigma_{a}$ is a parameter controlling the strength of the smoothness prior.

The ground truth anchor positions were $((0,0),(5.932,0),(3.0163,10.3079),(-0.6018,11.3658))$.

The estimated anchors were calculated to be $((0.4044,0.0429),(5.6435,-0.3461),(3.0475,10.5527)$,

$(-0.7488,11.4242))$. This gives a total Euclidean distance error of $0.5923 \mathrm{~m}$.

One section of the predefined path was obscured by a staircase. There the measurements had $6.52 \%$ missing data and $9.7826 \%$ of the data was considered to be outliers, according to our algorithm. In comparison to the whole data set we had $1.23 \%$ missing data and $3.3451 \%$ of the data was considered 
to be outliers. To further test our method, we conducted an experiment using ultra-wideband measurements in 3D with 6 anchors. Here we used the same number of iterations as before but using a tolerance of $T=0.2$ and the 5 receivers and 5 senders 3D minimal solver, the result is shown in Figure 3.

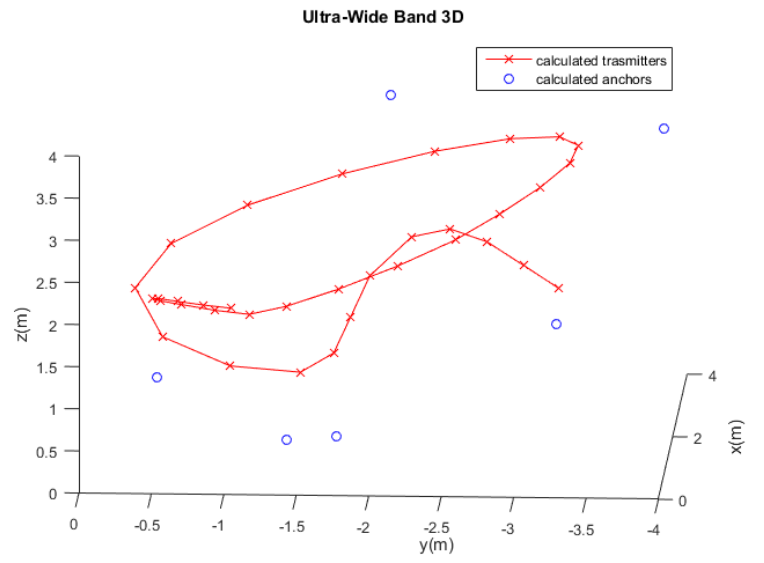

Fig. 3. This figure illustrates the estimated anchor and source positions.

\section{CONCLUSIONS}

In this paper we have constructed several systems for estimating receiver-sender node positions from measured receiversender distances in the presence of outliers and missing data. We propose several new methods for solving these problem. In particular we propose a novel hypothesis and test framework that efficiently finds initial estimates of the unknown parameters and combine such methods with efficient optimization techniques to obtain efficient, precise and robust systems. The proposed systems are evaluated against current state-of-theart methods on a large set of benchmark tests. Our proposed hypothesis and test framework is then further tested on real Wi-Fi and ultra-wideband measurements to solve time-ofarrival self-calibration and localization.

Looking at Figure 2, we can see that a reasonable estimation was calculated. The general predefined path shape was preserved and the anchor positions are reasonable with a total error of $0.5923 \mathrm{~m}$. Despite the path being obscured by stairs, the proposed method robustly and accurately estimated source positions. The experimental results demonstrates the robustness of the proposed method and how it can be effective in other areas as the errors in the estimations depend predominately on the accuracy of the distance measurements.

\section{REFERENCES}

[1] N.B. Priyantha, H. Balakrishnan, E. Demaine, and S. Teller, "Anchorfree distributed localization in sensor networks," in Proceedings of the 1 st international conference on Embedded networked sensor systems. ACM, 2003, pp. 340-341.

[2] G. Young and A.S. Householder, "Discussion of a set of points in terms of their mutual distances," Psychometrika, vol. 3, no. 1, pp. 19-22, 1938.

[3] Pratik Biswas, Tzu-Chen Lian, Ta-Chung Wang, and Yinyu Ye, "Semidefinite programming based algorithms for sensor network localization," ACM Trans. Sen. Netw., vol. 2, no. 2, pp. 188-220, May 2006.

[4] T. Eren, OK Goldenberg, W. Whiteley, Y.R. Yang, A.S. Morse, BDO Anderson, and PN Belhumeur, "Rigidity, computation, and randomization in network localization," in INFOCOM 2004. Twenty-third AnnualJoint Conference of the IEEE Computer and Communications Societies. IEEE, 2004, vol. 4, pp. 2673-2684.
[5] H. Stewénius, Gröbner Basis Methods for Minimal Problems in Computer Vision, Ph.D. thesis, Lund University, APR 2005.

[6] Yubin Kuang, Simon Burgess, Anna Torstensson, and Kalle Åström, “A complete characterization and solution to the microphone position selfcalibration problem," in The 38th International Conference on Acoustics, Speech, and Signal Processing, 2013.

[7] Simon Burgess, Yubin Kuang, and Kalle Åström, "Node localization in unsynchronized time of arrival sensor networks," in Proceedings of the 21 st International Conference on Pattern Recognition, 2012.

[8] Simon Burgess, Yubin Kuang, and Kalle Åström, "Pose estimation from minimal dual-receiver configurations," in Proceedings of the 21 st International Conference on Pattern Recognition, 2012.

[9] M. Pollefeys and D. Nister, "Direct computation of sound and microphone locations from time-difference-of-arrival data," in Proc. of International Conference on Acoustics, Speech and Signal Processing, 2008.

[10] M. Crocco, A. Del Bue, M. Bustreo, and V. Murino, "A closed form solution to the microphone position self-calibration problem," in 37th International Conference on Acoustics, Speech, and Signal Processing (ICASSP 2012), Kyoto, Japan, March 2012.

[11] Marco Crocco, Alessio Del Bue, and Vittorio Murino, "A bilinear approach to the position self-calibration of multiple sensors," Trans. Sig. Proc., vol. 60, no. 2, pp. 660-673, feb 2012.

[12] S. Thrun, "Affine structure from sound," in Proceedings of Conference on Neural Information Processing Systems (NIPS), Cambridge, MA, 2005, MIT Press.

[13] Y. Kuang, E. Ask, S. Burgess, and K. Åström, "Understanding toa and tdoa network calibration using far field approximation as initial estimate," in ICPRAM, 2012.

[14] M. A. Fischler and R. C. Bolles, "Random sample consensus: a paradigm for model fitting with applications to image analysis and automated cartography," Communications of the ACM, vol. 24, no. 6, pp. 381-95, 1981.

[15] Carl Eckart and Gale Young, "The approximation of one matrix by another of lower rank," Psychometrika, vol. 1, no. 3, pp. 211-218, 1936.

[16] T. Wiberg, "Computation of principal components when data are missing," in Proc. Second Symp. Computational Statistics, 1976.

[17] H. Aanaes, R. Fisker, K. Åström, and J.M. Carstensen, "Robust factorization," IEEE Trans. Pattern Analysis and Machine Intelligence, 2002.

[18] A. M. Buchanan and A. W. Fitzgibbon, "Damped newton algorithms for matrix factorization with missing data," in Proceedings of the 2005 IEEE Computer Society Conference on Computer Vision and Pattern Recognition (CVPR'05) - Volume 2 - Volume 02, Washington, DC, USA, 2005, CVPR '05, pp. 316-322, IEEE Computer Society.

[19] Q. Ke and T. Kanade, "Robust $L_{1}$ norm factorization in the presence of outliers and missing data by alternative convex programming," in Proc. Conf. Computer Vision and Pattern Recognition, 2005.

[20] A. Eriksson and A. Hengel, "Efficient computation of robust weighted low-rank matrix approximations using the $L_{1}$ norm," IEEE Trans. Pattern Analysis and Machine Intelligence, 2012.

[21] E. J. Candès, X. Li, Y. Ma, and J. Wright, "Robust principal component analysis?," Journal of ACM, 2009.

[22] Ravi Garg, Anastasios Roussos, and Lourdes Agapito, "Dense variational reconstruction of non-rigid surfaces from monocular video," in Computer Vision and Pattern Recognition (CVPR), 2013 IEEE Conference on. IEEE, 2013, pp. 1272-1279.

[23] C. Olsson and M. Oskarsson, "A convex approach to low rank matrix approximation with missing data," in Scandinavian Conf. on Image Analysis, 2011.

[24] Ricardo Cabral, Fernando De la Torre, Joao P. Costeira, and Alexandre Bernardino, "Unifying nuclear norm and bilinear factorization approaches for low-rank matrix decomposition," in Proc. Int. Conf. on Computer Vision, 2013.

[25] Lester W Mackey, Michael I Jordan, and Ameet Talwalkar, "Divideand-conquer matrix factorization," in Advances in Neural Information Processing Systems, 2011, pp. 1134-1142.

[26] Viktor Larsson, Carl Olsson, Erik Bylow, and Fredrik Kahl, "Rank minimization with structured data patterns," in ECCV, 2014.

[27] Fangyuan Jiang, Magnus Oskarsson, and Kalle Åström, "On the minimal problems of low-rank matrix factorization," in Proc. Conf. Computer Vision and Pattern Recognition, 2015. 